\title{
Similariton interactions in nonlinear graded-index waveguide amplifiers
}

\author{
Lei Wu (吴雷), ${ }^{1}$ Jie-Fang Zhang, ${ }^{1, *}$ Lu Li, $^{2}$ C. Finot, ${ }^{3}$ and K. Porsezian ${ }^{4}$ \\ ${ }^{1}$ Institute of Nonlinear Physics, Zhejiang Normal University, Jinhua, Zhejiang 321004, People's Republic of China \\ ${ }^{2}$ College of Physics and Electronics Engineering and Institute of Theoretical Physics, \\ Shanxi University, Taiyuan, Shanxi 030006, People's Republic of China \\ ${ }^{3}$ Institut Carnot de Bourgogne, UMR CNRS 5209, Université de Bourgogne, 9 avenue Alain Savary, 21078 Dijon, France \\ ${ }^{4}$ Department of Physics, School of Physical, Chemical and Applied Sciences, Pondicherry University, Pondicherry-605014, India
}

(Received 13 July 2008; published 5 November 2008)

\begin{abstract}
Self-similar propagation of optical beams inside a one-dimensional nonlinear graded-index waveguide amplifier is studied. By employing the lens-type and Husimi transformations, movable exact quasisoliton similaritons are obtained. Under certain conditions, these similaritons are well described by bright, Gaussian, and dark solitons on a compact parabolic background. Approximate but highly accurate analytical methods are developed to describe the interaction dynamics of exact quasisoliton similaritons.
\end{abstract}

DOI: 10.1103/PhysRevA.78.053807

PACS number(s): 42.81.Dp, 05.45.Yv, 42.65.Tg

\section{INTRODUCTION}

Self-similarity is a fundamental property that has been explored in many areas of physics such as hydrodynamics and quantum field theory [1]. Recently, the symmetry reduction method, which is based on the self-similarity of certain partial differential equations, has been widely applied to search for exact and asymptotic self-similar solutions in optical systems modeled by the well-known nonlinear Schrödinger equation (NLSE) [2-10]. These self-similar waves (optical similaritons) may be useful in real applications, since they can maintain their overall shapes but with their amplitudes and widths changing with the modulation of system parameters such as dispersion, nonlinearity, gain, and inhomogeneity. Similaritons also exist in fields such as plasmas and Bose-Einstein condensation [11-13].

One always deals with the following two types of similaritons in the context of nonlinear optics. The first are the asymptotic similaritons, which are mainly described by compact parabolic, Hermite-Gaussian, and hybrid functions [2-5,14-16]. These asymptotic similaritons exist under a wide range of system parameters, and hence have attracted much attention in recent years, especially the asymptotic parabolic similariton, which exhibits some interesting properties such as that it can be easily generated from arbitrary input waves and its stability is guaranteed even at a high power level [15]. The other type are the exact similaritons, described by exact soliton $[6-10,17]$ and quasisoliton solutions [18-20]. The existence of exact similaritons requires a delicate balance between system parameters. Note that the process of finding exact soliton similaritons is in essence the reduction of the original NLSE equation to the standard NLSE, and all of the methods can be unified by the generalized inverse scattering technique with variable spectral parameters [17]. The exact quasisoliton similariton (EQSS), however, is obtained by mapping the original NLSE equation into the standard NLSE with a stationary harmonic potential [18-20]. Therefore, exact multiple-soliton similariton solu-

\footnotetext{
*Corresponding author. jf_zhang@zjnu.cn
}

tions can be constructed by the inverse scattering technique, whereas exact multiple quasisoliton solutions are hard to find.

An important aspect of the similariton theory is the interaction dynamics. Recently, it was found that the collision of two asymptotic parabolic similaritons results in an oscillation, which further evolves into a train of dark solitons [16]; while the interaction of two exact soliton similaritons leads to the elastic collision or formation of a moleculelike bound state, depending crucially on the sign of the similariton phase chirp [9]. The interaction of multiple similaritons (more than two) were also studied numerically in loss- and dispersionmanaged fibers [21]; as for the EQSSs, it was shown that the initial chirps reduce their interaction [18]. However, the collision dynamics of multiple EQSSs has not yet been studied. In this paper, we are going to develop an analytical method to describe the interaction dynamics of multiple EQSSs in the context of a nonlinear graded-index waveguide amplifier.

\section{MODEL EQUATION AND THE EVOLUTION OF A SINGLE SIMILARITON}

We consider the propagation of an optical beam inside a nonlinear graded-index waveguide amplifier with refractive index $[8,22] n=n_{0}+n_{1} f(z) x^{2}+n_{2} I$, where $z$ is the propagation distance, $x$ is the spatial coordinate, and $I$ is the beam intensity. The first two terms describe the linear contribution to the refractive index and the last intensity-dependent term represents the Kerr nonlinearity (positive $n_{2}$ for self-focusing nonlinearity and negative $n_{2}$ for self-defocusing nonlinearity). Here we assume $n_{1}>0$, and $f(z)$ is the distributed parameter describing the inhomogeneity of the graded-index waveguide along the propagation direction. The gradedindex waveguide acts as a linear defocusing lens for $f(z)>0$ and a focusing lens for $f(z)<0$. With this kind of refractive index, one can control the collision of optical beams by choosing the appropriate inhomogeneity parameter $f$. The governing equation for the propagation of optical beams inside such a waveguide is the following (1+1)-dimensional NLSE: 


$$
i u_{z}+\frac{1}{2} u_{x x}+\sigma|u|^{2} u+f(z) \frac{x^{2}}{2} u=i \frac{g(z)}{2} u,
$$

where the beam envelope $u$, propagation distance $z$, spatial coordinate $x$, and gain parameter $g(z)$ are respectively normalized by $\left(k_{0}\left|n_{2}\right| L_{D}\right)^{-1 / 2}, L_{D}, w_{0}$, and $L_{D}^{-1}$, with the wave number $k_{0}=2 \pi n_{0} / \lambda$ at the input wavelength $\lambda$, the diffraction length $L_{D}=k_{0} w_{0}^{2}$, and the characteristic transverse scale $w_{0}=\left(2 k_{0}^{2} n_{1}\right)^{-1 / 4}$. The nonlinearity coefficient is $\sigma=\operatorname{sgn}\left(n_{2}\right)$ $= \pm 1$.

To obtain the exact self-similar solutions, we introduce the following lens-type transformation [20]:

$$
u(z, x)=\frac{\exp \left(\int_{0}^{z} g\left(z^{\prime}\right) / 2 d z^{\prime}\right)}{\sqrt{\ell(z)}} U(Z, X) \exp \left(i \frac{\ell_{z}}{2 \ell} x^{2}\right),
$$

where $\ell=\exp \left[\int_{0}^{z}-g\left(z^{\prime}\right) d z^{\prime}\right], X=x / \ell$, and $Z_{z}=\ell^{-2}$ with $Z(0)$ $=0$, and reduce the original equation (1) into the following standard NLSE with an additional harmonic potential:

$$
i U_{Z}+\frac{1}{2} U_{X X}+\sigma|U|^{2} U=K \frac{X^{2}}{2} U,
$$

where $K$ is determined by the self-similar condition

$$
K=\left(g^{2}-g_{z}-f\right) \ell^{4} .
$$

The virtue of the lens-type transformation is that we can obtain information about the original equation (1) via the investigation of Eq. (3). Specifically, when $K=0, U(Z, X)$ can be given explicitly by the bright $(\sigma=1)$ and dark soliton $(\sigma=-1)$ solutions, and the interaction of multiple similaritons can be obtained by the powerful inverse scattering technique. Here we are more interested in the case where $K$ is a positive constant, such that Eq. (3) possesses a localized stationary state solution $U(Z, X)=S(X) \exp (i \mu Z)$, where $\mu$ is the propagation constant. Note that this stationary state solution rests at the coordinate origin $X=0$. According to the Husimi transformation [23], a movable stationary state solution is possible. That is, if $U(Z, X)$ is a solution of Eq. (3), so is the following:

$$
U\left(Z, X-X_{0}\right) \exp \left[i\left(X_{0 Z}\left(X-X_{0}\right)+\Phi\right)\right]
$$

where the center-of-mass motion of the stationary state solution is given as

$$
X_{0 Z Z}+K X_{0}=0 \text {, }
$$

and the phase $\Phi=\int_{0}^{Z}\left(X_{0 Z}^{2}-K X_{0}^{2}\right) / 2 d Z+\Phi_{0}$. Note that the center-of-mass velocity of the stationary state is $X_{0 Z}$, which is just the coefficient of $X$ in the phase term. This is a unique property of the NLSE with constant coefficients.

Then the lens-type and Husimi transformations, as well as the self-similar condition, determine the exact self-similar evolution of the movable EQSS. It is readily shown that the amplitude and effective beamwidth of the EQSS are proportional to $\exp \left[\int_{0}^{z} g\left(z^{\prime}\right) d z^{\prime}\right]$ and $\exp \left[\int_{0}^{z}-g\left(z^{\prime}\right) d z^{\prime}\right]$, respectively, while its center of mass is $x_{0}=X_{0} \ell$, satisfying the Ehrenfest theorem:

$$
x_{0 z z}=f(z) x_{0} .
$$

It must be noted that the center-of-mass velocity of the EQSS is $x_{0 z}=X_{0 Z} / \ell-X_{0} g \ell$, which does not equal the coefficient of $x$ in the phase terms $X_{0 Z} / \ell$. The additional velocity $-X_{0} g \ell$ is caused by the coordinate transformation between the new variable $X$ and the old one $x$. Also note that the phase-front curvature of the EQSS is $\ell_{z} / 2 \ell$, which just equals one-half of the change rate of effective beamwidth $\ell$, this is also a unique property for the self-similar evolution of optical waves of Eq. (1).

\section{INTERACTION OF MULTIPLE SIMILARITONS}

The single-EQSS solution, which is directly related to the stationary state solution of Eq. (3), can be easily found by numerical methods; however, the multiple EQSSs are hard to retrieve. We hereafter present approximate but highly accurate descriptions of interaction dynamics of multiple EQSSs, which are composed of many single EQSSs.

\section{A. interaction of sech-type similaritons}

First, when the initial power of the optical beam is large, the nonlinearity is self-focusing $(\sigma=1)$, and $K$ is small, the envelope of the stationary state of Eq. (3) can be well described by the bright soliton solution:

$$
S=2 \eta \operatorname{sech}[2 \eta(X-Q)],
$$

where $2 \eta$ and $Q$ are the amplitude and center of mass of the bright soliton.

For a single bright soliton, the center-of-mass motion is determined by Eq. (6) with $X_{0}$ replaced by $Q$, which is the same as the formula in Ref. [24]. That is, the bright soliton behaves as a quasiparticle of mass 1, oscillating in a harmonic potential. Several approximate methods have been developed regarding the interaction of bright solitons. For example, Karpman and Solov'ev [25] used a perturbative analysis based on the inverse scattering technique to describe the interaction of two bright solitons of the standard NLSE; Gordon [26] derived the bright soliton interaction directly from the exact two-soliton function of the standard NLSE; while Anderson and Lisak [27] reduced the standard NLSE to two coupled NLSEs and obtained an expression for the interaction of two bright soliton based on the variational technique. Later, Gerdjikov et al. [28] found that the interaction of $N$ bright solitons leads to a complex Toda lattice with $N$ nodes, where the NLSE can have an external potential. However, one of the validity conditions of the above methods is that the relative velocity between the two bright solitons is small everywhere, corresponding to slow collisions, which could lead to energy transformation between bright solitons. On the contrary, when two bright solitons collide with a relative large velocity, their shapes remain intact but their centers and phases suffer a shift. To describe this shift, Scharf and Bishop [29] mapped the system to a nonintegrable many-particle dynamics with an effective Hamiltonian by a collective-coordinate approximation. The validity of the effective Hamiltonian is under the assumption that threesoliton collisions as well as two-soliton collisions with van- 
(a)

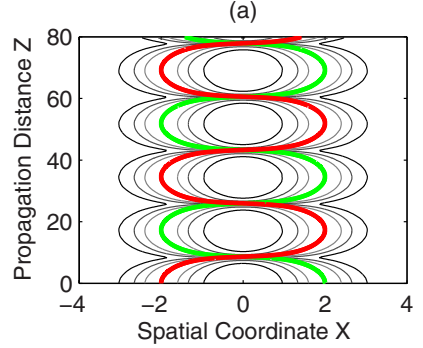

(b)

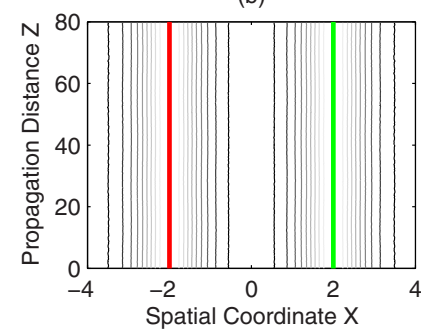

FIG. 1. (Color online) Evolution of the amplitude of $U(Z, X)$ for (a) in-phase collision and (b) out-of-phase interaction of two bright solitons. The initial velocity of bright solitons is zero; the other parameters are $\eta=1, \quad Q_{2}(0)=-Q_{1}(0)=2$, and $K=8 \eta^{3} \operatorname{sech}^{2}\left[2 \eta Q_{1}(0)\right] \tanh \left[2 \eta Q_{1}(0)\right] / Q_{1}(0)$.

ishing relative velocity can be neglected. Fortunately, the two cases happen rarely. Recently, the Hamiltonian was employed to study the chaotic collision between three bright solitons of Eq. (3) in the context of Bose-Einstein condensation, where the numerical results from the effective Hamiltonian agrees well with direct numerical simulations of the Gross-Pitaevskii equation [30]. In this paper, we introduce a Hamiltonian for the center-of-mass motion of an arbitrary number of bright solitons in the framework of Eq. (3) as

$$
\begin{aligned}
H= & \sum_{i=1}^{N}\left(\frac{P_{i}^{2}}{2 \eta_{i}}+\frac{\eta_{i} K Q_{i}^{2}}{2}\right)-\sum_{1 \leqslant i<j \leqslant N} \cos \left(\Delta \Phi_{i j}\right) \\
& \times 2 \eta_{i} \eta_{j}\left(\eta_{i}+\eta_{j}\right) \operatorname{sech}^{2}\left(\frac{2 \eta_{i} \eta_{j}}{\eta_{i}+\eta_{j}}\left(Q_{i}-Q_{j}\right)\right),
\end{aligned}
$$

where $P_{i}$ and $Q_{i}$ are, respectively, the the momentum and center of mass of the $i$ th soliton, and $\Delta \Phi_{i j}$ is the initial phase difference between the $i$ th and $j$ th solitons, with $\Delta \Phi_{i j}=0$ and $\pi$ corresponding to the in-phase and out-phase collisions between the $i$ th and $j$ th solitons, respectively.

Now we consider the interaction of two identical solitons, namely, $\eta_{1}=\eta_{2}=\eta$. From the Hamiltonian (9), it is readily shown that the center-of-mass motion of each soliton is governed by the following equations:

$$
\begin{aligned}
Q_{1 Z Z}= & -K Q_{1}-8 \eta^{3} \cos (\Delta \Phi) \operatorname{sech}^{2}\left[\eta\left(Q_{1}-Q_{2}\right)\right] \\
& \times \tanh \left[\eta\left(Q_{1}-Q_{2}\right)\right], \\
Q_{2 Z Z}= & -K Q_{2}+8 \eta^{3} \cos (\Delta \Phi) \operatorname{sech}^{2}\left[\eta\left(Q_{1}-Q_{2}\right)\right] \\
& \times \tanh \left[\eta\left(Q_{1}-Q_{2}\right)\right],
\end{aligned}
$$

where the first terms in the right-hand sides of Eq. (10) stand for the linear restoring force introduced by the external potential $K X^{2} / 2$, which dominates at large relative distance; while the second terms in the right-hand sides of Eq. (10) represent the nonlinear interaction force, which work at intermediate relative distance. The nonlinear force is attractive for in-phase collisions and repulsive for out-phase collisions.

Figure 1 shows two typical interaction sceneries of two identical bright solitons, where the direct numerical simulation results (black lines, contour plot of the amplitude) of Eq. (3) agree well with the numerical integration results (red and green lines, center of mass of each bright soliton) of Eq. (10).
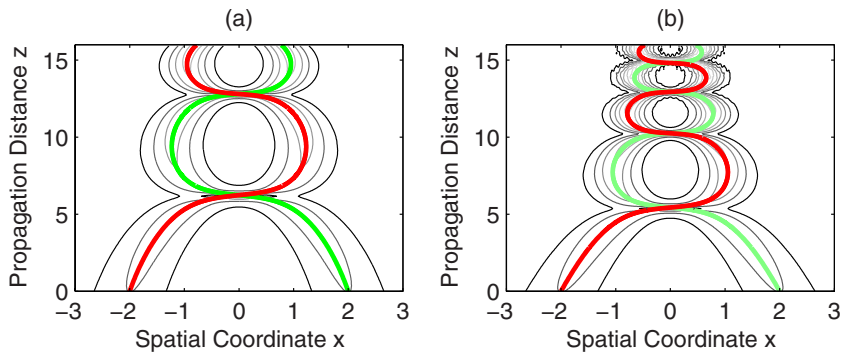

(c)

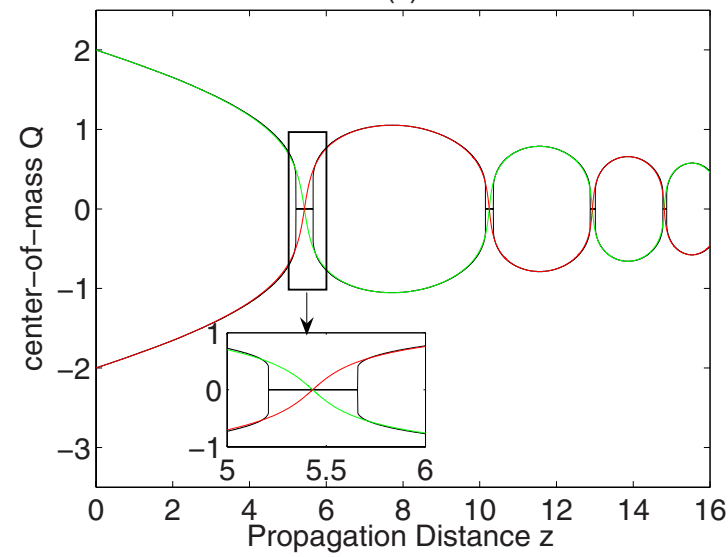

FIG. 2. (Color online) (a), (b) Evolution of the amplitude of $u(z, x)$ for the in-phase collision of two EQSSs. (c) Comparison between the trajectory of center of mass between the direct numerical simulation of Eq. (1) (black lines) and Eq. (11) (colored lines), where the parameters are the same as those used in (b). The inhomogeneity parameter $f=g^{2}-K \exp (4 g z)$, gain parameters $g$ are respectively 0.05 and 0.08 for (a) and (b), and the other parameters are the same as in Fig. 1(a).

When the two solitons are in phase, they collide periodically [Fig. 1(a)]; when the two solitons are out of phase, they propagate with constant distance when $Q_{i Z Z}(0)$ and $Q_{i Z}(0)$ are zero, where $i=1,2$ [Fig. 1(b)]. This is due to the balance between the linear restoring force and the nonlinear repulsive force.

The interaction scenario of two identical EQSSs will be different when the lens-type and Husimi transformations as well as the self-similar condition are taken into account. Such a difference is caused by the transformation between $Z$ and $z, X$ and $x$ [31]. We first take the initial condition of Eq. (1) as $u(0, x)=U(0, X) \exp \left[-i g(0) x^{2} / 2\right]$ with $U(0, X)$ being the initial conditions used in Fig. 1(a), and the gain parameter $g$ is constant for the example. When $g$ is a positive constant, we have $z=\ln (2 g Z+1) / 2 g$, which implies that the collision period gets shorter and shorter as $z$ and $g$ increase (Fig. 2). On the other hand, when $g$ is a negative constant, we have $Z=[1-\exp (-2|g| z)] / 2|g|$. Quite interestingly, the new propagation distance $Z$ now has an upper-bound value $1 / 2|g|$, which means that for some value of $g$, the periodic collision will not appear. From Fig. 1(a) we see that the two solitons collide at $Z \approx 8-9$ for the first time and at $Z$ $\approx 25-26$ for the second time, so the two corresponding EQSSs collide only once when $g=-0.03$ and then separate [Fig. 3(a)], this is because the upper-bound value is $Z_{\max }$ $\approx 16.7$, which is larger than 9 but smaller than 25 ; similarly, 
(a)

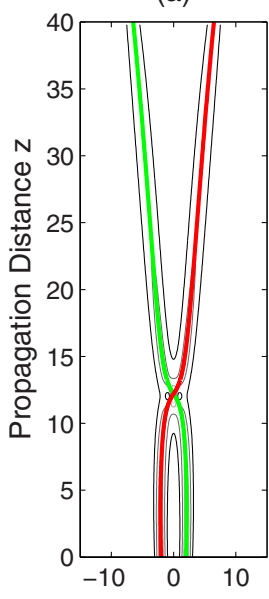

(b)

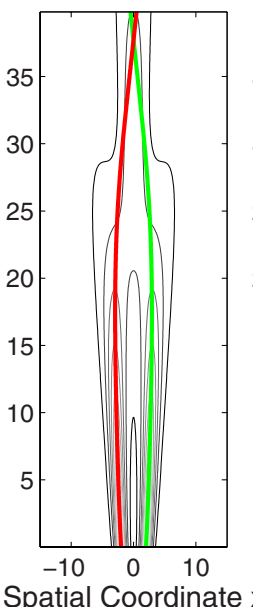

(c)

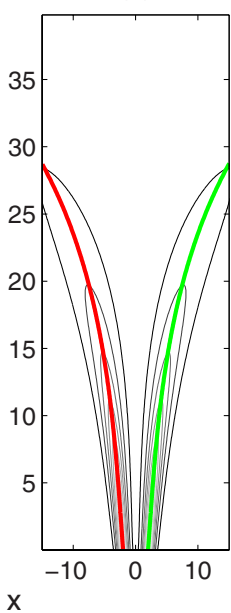

FIG. 3. (Color online) Evolution of the amplitude of $u(z, x)$ for the in-phase interaction of two EQSSs. The inhomogeneity parameter $f=g^{2}-K \exp (4 g z)$, gain parameters $g$ are respectively -0.03 , -0.057 , and -0.08 for (a), (b), and (c), and the other parameters are the same as in Fig. 1(a).

the two EQSSs collide once but now form a moleculelike bound state when $g=-0.057$ such that $Z_{\max } \approx 8.8$ [Fig. 3(b)]; while when $g=-0.08$ such that $Z_{\max }=6.25$, the two EQSSs did not collide but separated [Fig. 3(c)]. Note that the underlying dynamics for the formation of the moleculelike bound state is that, when the original propagation distance $z$ varies a lot, the transformed propagation distance $Z$ only changes a little, and it happens to be in the region where two stationary state solitons collide. To achieve this, the amplification parameter $g$ should be negative; therefore, the formation of the moleculelike bound state depends crucially on the phasefront curvature, but not the initial phase-front curvature (also known as chirp in the context of temporal optics [9]). When $U(0, X)$ is taken as the initial condition used in Fig. 1(b), the results are quite simple: the two corresponding EQSSs either approach each other when $g$ is positive or separate when $g$ is negative (Fig. 4). For other forms of amplification parameters and corresponding inhomogeneity parameters, similar situations will happen.

To further understand the interaction dynamics of two identical EQSSs, we present here the governing equation for (a)

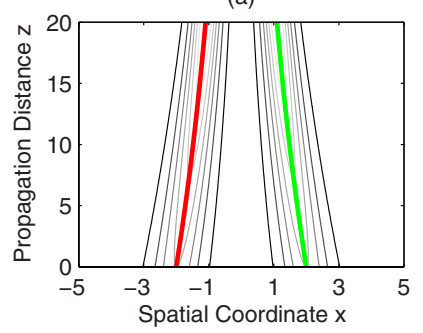

(b)

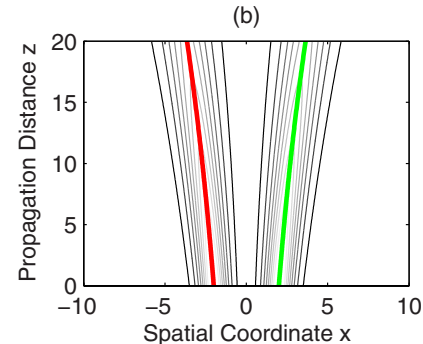

FIG. 4. (Color online) Evolution of the amplitude of $u(z, x)$ for the out-of-phase interaction of two EQSSs. The inhomogeneity parameter $f$ is $g^{2}-K \exp (4 g z)$, gain parameters $g$ are respectively 0.03 and -0.03 for (a) and (b), and the other parameters are the same as in Fig. 1(b). their centers of mass $x_{1}$ and $x_{2}$ in the original coordinates $x$ and $z$ (while the changes of amplitudes and widths are given by the lens-type transformation):

$$
\begin{aligned}
x_{1 z z}= & f(z) x_{1}-\frac{8 \eta^{3}}{\ell(z)^{3}} \operatorname{sech}^{2}\left(\frac{\eta\left(x_{1}-x_{2}\right)}{\ell(z)}\right) \\
& \times \tanh \left(\frac{\eta\left(x_{1}-x_{2}\right)}{\ell(z)}\right) \cos (\Delta \Phi), \\
x_{2 z z}= & f(z) x_{2}+\frac{8 \eta^{3}}{\ell(z)^{3}} \operatorname{sech}^{2}\left(\frac{\eta\left(x_{1}-x_{2}\right)}{\ell(z)}\right) \\
& \times \tanh \left(\frac{\eta\left(x_{1}-x_{2}\right)}{\ell(z)}\right) \cos (\Delta \Phi) .
\end{aligned}
$$

Such an equation is obtained by substituting the coordinate transformation used in the lens-type transformation into Eq. (10) and considering the fact that $x_{0}=Q_{0} \ell$. It should be noted that the initial velocity of each similariton is $x_{i z}(0)$ $=Q_{i Z}(0) / \ell-Q_{i}(0) g \ell$. The validity of this equation has been confirmed in Figs. 2-4, where one can clearly find that the numerical results (colored lines) of Eq. (11) agree well with the direct numerical simulations of Eq. (1), except for the situation when the two bright similaritons collide [Fig. 2(c)]. This is caused by the numerical error in determining the center of mass of two sech-type similaritons from the direct numerical simulation data of Eq. (1) when their profiles are almost overlapped.

Obviously, the above analysis can be easily extended to study the interaction dynamics of multiple EQSSs. Specifically, when the EQSSs have the same amplitude and width, their center-of-mass motion can be simply given:

$$
\begin{aligned}
x_{i z z}= & f(z) x_{i}-\frac{8 \eta^{3}}{\ell(z)^{3}} \sum_{i \neq j} \operatorname{sech}^{2}\left(\frac{\eta\left(x_{i}-x_{j}\right)}{\ell(z)}\right) \\
& \times \tanh \left(\frac{\eta\left(x_{i}-x_{j}\right)}{\ell(z)}\right) \cos \left(\Delta \Phi_{i j}\right),
\end{aligned}
$$

where $i=1,2, \ldots, N$ is the EQSS index, with $N$ being the total number of EQSSs.

Figure 5 shows the trajectory of three EQSSs with the initial condition $u(0, x)=\sum_{i}^{3} 2 \operatorname{sech}\left[2\left(x+x_{i}\right)\right] \exp \left(i \Phi_{i}\right)$, where the numerical results (color lines) of Eq. (12) agree well with the direct numerical simulation (black lines) of Eq. (1). Note that, unlike the interaction of two EQSSs, where their centerof-mass motions are regular, the interaction of multiple EQSSs could lead to the emergence of chaos [30]. Chaos is a fascinating character of nonlinear systems. In nonlinear optics, chaos has also been reported in the coupled NLSE for the collision of vector solitons [32] and in massive multichannel optical fiber communication systems with the effect of delayed Raman response [33]. Here the presence of $f(z)$ and $\ell(z)$ in Eq. (12), as well as the relative phase difference, provide a method to control the chaotic behavior of multiple EQSSs. For example, when three identical EQSSs are initially located at $Q, 0,-Q$ with initial phase $0, \pi, 0$ and $K$ $=8 \eta^{3}\left[\operatorname{sech}^{2}(\eta Q) \tanh (\eta Q)-\operatorname{sech}^{2}(2 \eta Q) \tanh (2 \eta Q)\right] / Q$, three EQSS bound states could emerge. On the other hand, since 
(a)

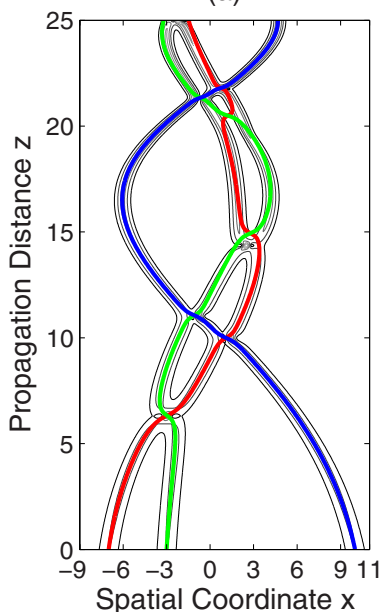

(b)

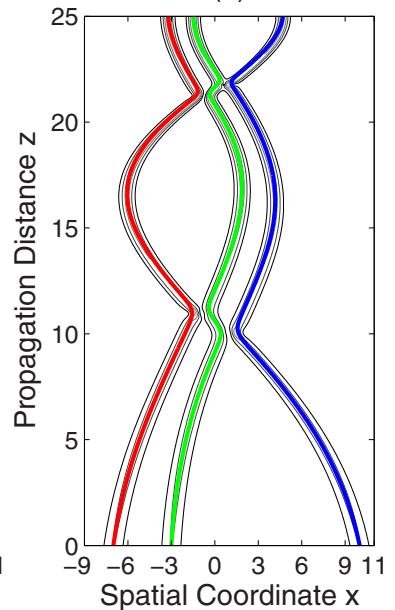

FIG. 5. (Color online) Evolution of the amplitude $u(z, x)$ of three identical EQSSs are initially located at $-7,-3$, and 10 . The inhomogeneity parameter is $f=g^{2}-K \exp (4 g z)$ with the gain parameter $g$ being 0.03 . The initial phase is $\Phi_{1}=\Phi_{2}=\Phi_{3}=0$ in (a) and $\Phi_{1}=0, \Phi_{2}=\pi, \Phi_{3}=0$ in (b).

the chaotic behavior stems from periodic collisions, we can choose negative $g$ and corresponding $f$ to control the collision times of the multiple EQSSs, as demonstrated in Fig. 3(c).

\section{B. Interference of Gaussian-type similaritons}

Second, when the initial power of the optical beam is weak such that the nonlinearity is negligible, the stationary state solution of Eq. (3) can be well described by the Hermite-Gaussian function. Here we consider only the Gaussian function, since other high-order stationary solutions can be handled in the same way:

$$
U_{0}(Z, X)=A_{0} \exp \left(-\frac{X^{2}}{2 W_{0}^{2}}-i \mu Z\right),
$$

where $W_{0}$ is the effective beamwidth at equilibrium, satisfying $K=1 / W_{0}^{4}$, and $\mu=1 / 2 W_{0}^{2}$ is the propagation constant.

When the effective beamwidth strays from the equilibrium value $W_{0}$, or the Gaussian beam has initial phase-front curvature, the general self-similar Gaussian beam of Eq. (3) can be given:

$$
U(Z, X)=A \exp \left(-\frac{X^{2}}{2 W^{2}}\right) \exp \left[i\left(\frac{W_{Z}}{2 W} X^{2}-\int_{0}^{Z} \frac{1}{W^{2}} d Z\right)\right] .
$$

Here the effective beamwidth is $W=W_{0}+W_{p}$, where the periodically changing width $W_{p}$ is determined by $W_{p Z Z}$ $=4 K W_{p}$ [34], and the amplitude of the Gaussian beam $A$ satisfies the conservation law of total beam energy as $P$ $=\sqrt{\pi} A^{2} W$.

Therefore, Eq. (14), the lens-type transformation, selfsimilar condition, and Husimi transformation describe the exact self-similar evolution of a Gaussian beam of the original equation (1). Note that the effective beamwidth of the
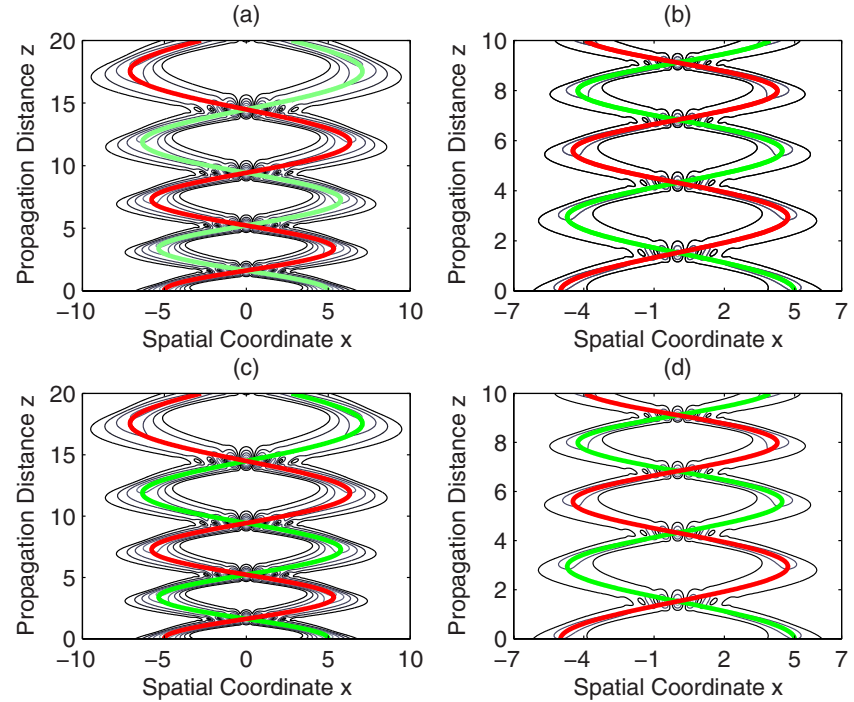

FIG. 6. (Color online) In-phase interference of two Gaussian EQSSs. The inhomogeneity parameter is $f=g^{2}-K \exp (4 g z)$ with the gain parameter $g$ being -0.02 for (a) and (c) and 0.02 for (b) and (d). The nonlinearity is self-focusing in (a) and (b) and selfdefocusing in (c) and (d). The initial positions of the EQSSs are -5 and $5, A(0)=0.1, W(0)=K=1$, and $W_{Z}(0)=1$.

EQSS is now proportional to $\exp \left[\int_{0}^{z} g\left(z^{\prime}\right) d z^{\prime}\right] W(Z)$. Since in the low-intensity approximation the NLSE (3) is approximately linear, the interaction of two EQSSs is quite simple: their center-of-mass motion is determined by $x_{i z z}=f x_{i}$, and their effective beamwidth varies as $W_{i 0} \ell+w_{i}$, with $w_{i}$ determined by $w_{i z z}=4 f w_{i}$; when they collide, strong interference structures appear [35]. One can see that there are no major difference between the self-focusing and self-defocusing nonlinearity (Fig. 6), since for the Gaussian-type similaritons both the focusing and defocusing nonlinearity are negligible.

\section{Interaction of tanh-type similaritons on a compact parabolic background}

Finally, when the initial power of the optical beam is large, nonlinearity is self-defocusing $(\sigma=-1)$ and the stationary state solution of Eq. (3) can be described by either the compact parabolic solution [20]

$$
U_{0}(Z, X)=A_{0} \sqrt{1-\frac{X^{2}}{W_{0}^{2}}} \exp \left(i \mu_{0} Z\right)
$$

or the dark soliton solution on the compact parabolic background $U_{0}(Z, X)$,

$$
U_{d}(Z, X)=U_{0}(Z, X) \tanh (A X),
$$

at $|X| \leqslant W_{0}=\sqrt{2 \mu_{0} / K}$ and $U(Z, X)=0$ otherwise, where $A_{0}$ $=\sqrt{\mu_{0}}$, and the propagation constant $\mu_{0}$ is related to the total beam energy as $P=\sqrt{32 \mu_{0}^{3} / 9 K}$.

Therefore Eq. (15) or Eq. (16), together with the lens-type transformation, self-similar condition, and Husimi transformation, describe the exact self-similar evolution of a parabolic beam or dark soliton on the compact parabolic background of the original equation (1). It is readily shown that 
(a)

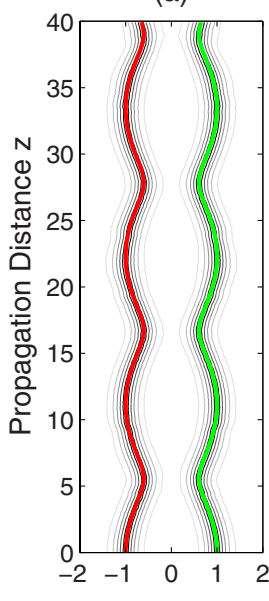

(b)

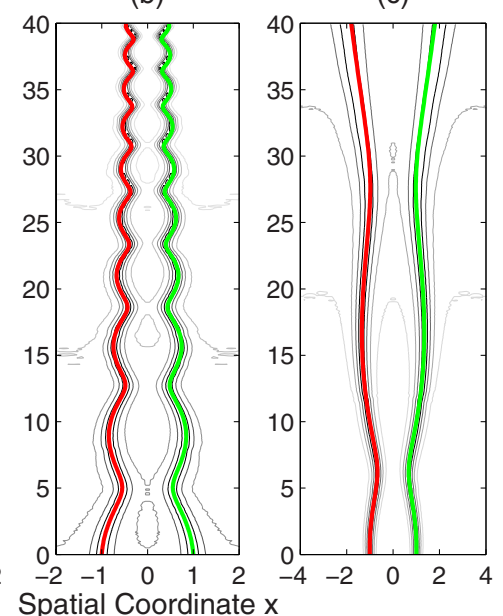

FIG. 7. (Color online) Interaction of two dark solitons on the self-similar compact parabolic background. The inhomogeneity parameter is $f=g^{2}-K \exp (4 g z)$ with the gain parameter $g$ being 0 for (a), 0.02 for (b), and -0.02 for (c). The initial positions of the dark solitons are -1 and $1, A_{0}=3$, and $W_{0}=15$.

the amplitude and effective beamwidth of one compact parabolic similariton are proportional to $\exp \left[\int_{0}^{z} g\left(z^{\prime}\right) d z^{\prime}\right]$ and $\exp \left[\int_{0}^{z}-g\left(z^{\prime}\right) d z^{\prime}\right]$, respectively. However, the interaction of two parabolic EQSSs is much more complicated than that of Gaussian EQSSs, where the governing equations are approximately linear. Numerical simulations show that, when the two parabolic EQSSs are separated by a distance much larger than their widths, their interaction scenario can be approximated by their linear superposition for the first several collision periods, since the nonlinearity does not have much time to convert the interference pattern to dark solitons. On the contrary, when their relative distance is much smaller than their width, their strong interaction will quickly lead to the formation of dark solitons. Here we consider the special case that the initial condition of Eq. (3) takes the form

$$
U(0, X)=U_{0}(0, X) \tanh \left[A_{0}\left(X-Q_{1}\right)\right] \tanh \left[A_{0}\left(X-Q_{2}\right)\right],
$$

to study the interaction of two dark solitons on a compact parabolic background.

It is well known that, when there is only one dark soliton on a compact parabolic background, that is,

$$
U=U_{0}\left\{\cos (\phi) \tanh \left[A_{0} \cos (\phi)(X-Q)\right]+i \sin (\phi)\right\},
$$

the center-of-mass motion of the dark soliton is determined by $Q_{Z Z}+K Q / 2=0$ and $Q_{Z}=A_{0} \sin (\phi)$ with $|\phi| \leqslant \pi / 2$, provided that the oscillation amplitude $\max (Q)$ is much smaller than the width of the parabolic region [36]. That is, the dark soliton behaviors as a quasiparticle of mass 2 , oscillating on a compact parabolic background; when taking into account their interactions, we suppose that the interaction forces, which are repulsive, have the same expression as that for bright solitons (9). Therefore, in the small-oscillation-
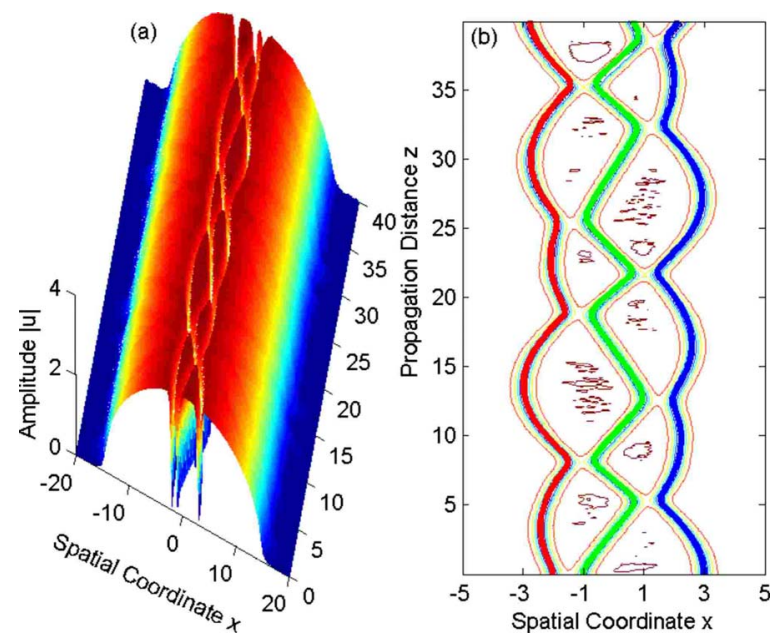

FIG. 8. (Color online) Interaction of three dark solitons on a compact parabolic background: (a) amplitude and (b) trajectory. The inhomogeneity parameter is $f=-K$, and the gain parameter is $g=0$. The initial positions of the dark solitons are $-2,-1$, and 3 , $A_{0}=3$, and $W_{0}=15$.

amplitude approximation where $Q_{1}, Q_{2} \ll W_{0}$, the governing equations for the centers of mass of the two dark solitons are

$$
\begin{aligned}
& Q_{1 Z Z}=-\frac{K}{2} Q_{1}+2 A_{0}^{3} \operatorname{sech}^{2}\left[A_{0}\left(Q_{1}-Q_{2}\right)\right] \tanh \left[A_{0}\left(Q_{1}-Q_{2}\right)\right], \\
& Q_{2 Z Z}=-\frac{K}{2} Q_{2}+2 A_{0}^{3} \operatorname{sech}^{2}\left[A_{0}\left(Q_{2}-Q_{1}\right)\right] \tanh \left[A_{0}\left(Q_{2}-Q_{1}\right)\right],
\end{aligned}
$$

while the initial condition (17) evolves as $U(Z, X)$ $=U_{0} \Pi_{i=1}^{2}\left\{\cos \left(\phi_{i}\right) \tanh \left[A_{0} \cos \left(\phi_{i}\right)\left(X-Q_{i}\right)\right]+i \sin \left(\phi_{i}\right)\right\}, \quad$ with $\sin \left(\phi_{i}\right)=Q_{i Z} / A_{0}$ and $\left|\phi_{i}\right| \leqslant \pi / 2$.

As for the interaction of $N$ bright solitons, we can get the governing equation for the center-of-mass motion of $N$ dark solitons on a self-similar compact parabolic background in the original spatial and temporal coordinates $x$ and $z$ :

$$
\begin{aligned}
x_{i z z}= & \frac{f(z)}{2} x_{i}+\frac{2 A_{0}^{3}}{\ell(z)^{3}} \sum_{i \neq j} \operatorname{sech}^{2}\left(\frac{A_{0}\left(x_{i}-x_{j}\right)}{\ell(z)}\right) \\
& \times \tanh \left(\frac{A_{0}\left(x_{i}-x_{j}\right)}{\ell(z)}\right),
\end{aligned}
$$

where $x_{i}$ should satisfy the small-oscillation-amplitude approximation $x_{i} \ll W_{0} \ell$.

The validity of Eq. (20) has been confirmed by numerical simulations (Figs. 7 and 8), provided that the initial dark solitons do not overlap too much. From the numerical simulations, we found that the amplitude and width of the compact parabolic background are proportional to $\exp \left[\int_{0}^{z} g\left(z^{\prime}\right) d z^{\prime}\right]$ and $\exp \left[\int_{0}^{z}-g\left(z^{\prime}\right) d z^{\prime}\right]$, respectively, while the depth (minimum value of the intensity) and the effective beamwidth of the each dark soliton are proportional to $(C / \ell)^{2}$ and $\ell / C$, respectively, where $C=\cos \left(\phi_{i}\right)$ $=\sqrt{1-\left(x_{i z} \ell+x_{i} g \ell\right)^{2} / A_{0}^{2}}$, provided that the dark similaritons do not overlap too much. It is quite interesting that, as one can 
see from Eq. (19), the repulsive interaction force between dark solitons first increases as the relative distance (say, $\left.\left|Q_{1}-Q_{2}\right|\right)$ decreases, and then decreases to zero as the relative distance approaches zero. This is not physical since the repulsion should be increasing as the relative distance decreases. However, we can see in Fig. 8(b) that, even when the relative distances of dark solitons are very small, their trajectories obtained from Eq. (19) agree well with the direct numerical simulation of Eq. (1). This needs further investigation. Additional numerical simulations show that the interaction forces lead to chaotic behavior of multiple dark solitons propagating on a self-similar compact parabolic ground, like that of the bright similaritons.

\section{CONCLUSION}

In conclusion, we have obtained the self-similar evolution of optical beams inside the nonlinear graded-index waveguide amplifier. We have found that, under certain conditions, the optical similaritons can be described by the bright soliton solution, Hermite-Gaussian function, and dark soliton solution on a compact parabolic ground. The evolutions of the amplitudes and widths of exact optical similaritons are given by the lens-type transformation, while their center-ofmass motion is determined by approximate but highly accurate analytical methods. Specifically, we have investigated the interaction of two identical bright similaritons in detail, and explained why they can form a moleculelike bound state. We hope that our results will be very useful in initiating experimental verification.

\section{ACKNOWLEDGMENTS}

J.-F.Z. and L.W. acknowledge support of the National Natural Science Foundation of China (Grant No. 10672147). L.L. acknowledges support of the Provincial Natural Science Foundation of Shanxi (Grant No. 2007011007). K.P. wishes to thank the DST-Ramanna program, IFCPAR (Grant No. IFC/3504-F/2005/2064), DAE-BRNS, CSIR, Government of India, for financial support through research projects.
[1] G. I. Barenblatt, Scaling, Self-Similarity, and Intermediate Asymptotics (Cambridge University Press, Cambridge, U.K., 1996).

[2] M. E. Fermann, V. I. Kruglov, B. C. Thomsen, J. M. Dudley, and J. D. Harvey, Phys. Rev. Lett. 84, 6010 (2000).

[3] V. I. Kruglov, A. C. Peacock, J. M. Dudley, and J. D. Harvey, Opt. Lett. 25, 1753 (2000).

[4] V. I. Kruglov, A. C. Peacock, J. D. Harvey, and J. M. Dudley, J. Opt. Soc. Am. B 19, 461 (2002); V. I. Kruglov and J. D. Harvey, ibid. 23, 2541 (2006).

[5] Shihua Chen, Lin Yi, Dong-Sheng Guo, and Peixiang Lu, Phys. Rev. E 72, 016622 (2005).

[6] V. I. Kruglov, A. C. Peacock, and J. D. Harvey, Phys. Rev. Lett. 90, 113902 (2003).

[7] V. M. Pérez-García, P. J. Torres, and V. V. Konotop, Physica D 221, 31 (2006).

[8] S. A. Ponomarenko and G. P. Agrawal, Phys. Rev. Lett. 97, 013901 (2006); Opt. Lett. 32, 1659 (2007).

[9] S. A. Ponomarenko and G. P. Agrawal, Opt. Express 15, 2963 (2007).

[10] Juanfen Wang, Lu Li, and Suotang Jia, J. Opt. Soc. Am. B 25, 1254 (2008).

[11] L. Wu, Q. Yang, and J. F. Zhang, J. Phys. A 39, 11947 (2006).

[12] J. K. Xue, J. Phys. B 38, 3841 (2005).

[13] L. Wu, J. F. Zhang, and L. Li, New J. Phys. 9, 69 (2007); L. Wu, L. Li, G. Chen, Q. Tian, and J. F. Zhang, ibid. 10, 023021 (2008).

[14] Guoqing Chang, H. G. Winful, A. Galvanauskas, and T. B. Norris, Phys. Rev. E 72, 016609 (2005).

[15] J. M. Dudley, C. Finot, D. J. Richardson, and G. Millot, Nat. Phys. 3, 597 (2007).

[16] C. Finot and G. Millot, Opt. Express 13, 5825 (2005); 13, 7653 (2005).

[17] V. N. Serkin and A. Hasegawa, Phys. Rev. Lett. 85, 4502 (2000); V. N. Serkin, A. Hasegawa, and T. L. Belyaeva, ibid.
98, 074102 (2007).

[18] Shiva Kumar and A. Hasegawa, Opt. Lett. 22, 372 (1997).

[19] Y. Ozeki and T. Inoue, Opt. Lett. 31, 1606 (2006).

[20] L. Wu, J. F. Zhang, L. Li, Q. Tian, and K. Porsezian, Opt. Express 16, 6352 (2008).

[21] S. A. Ponomarenko and G. P. Agrawal, J. Opt. Soc. Am. B 25, 983 (2008).

[22] S. Raghavan and G. P. Agrawal, Opt. Commun. 180, 377 (2000).

[23] H. H. Chen and C. S. Liu, Phys. Rev. Lett. 37, 693 (1976).

[24] C. Hernandez Tenorio, E. Villargan Vargas, V. N. Serkin, M. Agüero Granados, T. L. Belyaeva, R. Peña Moreno, and L. Morales Lara, Quantum Electron. 35, 778 (2005).

[25] V. I. Karpman and V. V. Solov'ev, Physica D 3, 487 (1981).

[26] J. P. Gordon, Opt. Lett. 8, 596 (1983).

[27] D. Anderson and M. Lisak, Opt. Lett. 11, 174 (1986).

[28] V. S. Gerdjikov, D. J. Kaup, I. M. Uzunov, and E. G. Evstatiev, Phys. Rev. Lett. 77, 3943 (1996); V. S. Gerdjikov, B. B. Baizakov, M. Salerno, and N. A. Kostov, Phys. Rev. E 73, 046606 (2006).

[29] Rainer Scharf and A. R. Bishop, Phys. Rev. A 46, R2973 (1992).

[30] A. D. Martin, C. S. Adams, and S. A. Gardiner, Phys. Rev. Lett. 98, 020402 (2007).

[31] L. Wu and J. F. Zhang, Chin. Phys. Lett. 14, 1471 (2007).

[32] J. Yang and Y. Tan, Phys. Rev. Lett. 85, 3624 (2000); R. H. Goodman and R. Haberman, Phys. Rev. E 71, 056605 (2005).

[33] A. Peleg, Phys. Lett. A 360, 533 (2007); Y. Chung and A. Peleg, Phys. Rev. A 77, 063835 (2008).

[34] C. J. Pethick and H. Smith, Bose-Einstein Condensation in Dilute Gases (Cambridge University Press, Cambridge, U.K., 2001).

[35] H. F. Zhang, L. Li, and S. T. Jia, Phys. Rev. A 76, 043833 (2007).

[36] T. Busch and J. R. Anglin, Phys. Rev. Lett. 84, 2298 (2000). 